\title{
Anabases
}

ANABASES Traditions et réceptions de l'Antiquité

$11 \mid 2010$

Varia

\section{Wilhelm Weber et les Res Gestae Divi Augusti. La postérité d'un livre complexe}

John Scheid

\section{(2) OpenEdition}

Journals

Édition électronique

URL : http://journals.openedition.org/anabases/803

DOI : 10.4000/anabases.803

ISSN : 2256-9421

Éditeur

E.R.A.S.M.E.

Édition imprimée

Date de publication : 1 mars 2010

Pagination : 107-121

ISSN : 1774-4296

\section{Référence électronique}

John Scheid, «Wilhelm Weber et les Res Gestae Divi Augusti. La postérité d'un livre complexe »

Anabases [En ligne], 11 | 2010, mis en ligne le 01 mars 2013, consulté le 20 octobre 2019. URL : http:// journals.openedition.org/anabases/803 ; DOI : 10.4000/anabases.803

(c) Anabases 


\title{
Wilhelm Weber \\ et les Res Gestae Divi Augusti. La postérité d'un livre complexe
}

JOHN SCHEID

\author{
"A half truth is more dangerous (and wicked) \\ than a plain down-right invention." \\ Tennyson d'après W.M. Ramsay
}

$\mathrm{E}_{\mathrm{N}}$ 1929, W.M. RAMSAY ${ }^{1}$ citait dans une réponse à une attaque le poète Alfred Tennyson qui considérait qu'une demi-vérité est plus dangereuse et vicieuse qu'une invention pure et simple. Pour démontrer la justesse de cette formule, on peut prendre le texte qui a été à l'origine de la polémique dont la citation est extraite, les Res Gestae Diui Augusti. Il s'agit d'un texte qui se présente comme l'autobiographie politique d'Auguste, publiée après sa mort en 14 ap. J.-C. Des Res gestae qui avaient été inscrites sur deux stèles placées devant le mausolée d'Auguste à Rome, nous n'avons gardé aucune trace. Il existe, en revanche des copies retrouvées en Galatie, qui sont inégalement conservées. À Ankara, le texte complet mais parfois mutilé ou effacé du texte latin et de sa traduction grecque est conservé. Une partie de la version grecque a été retrouvée à Apollonia de Pisidie, et une version latine fragmentaire à Antioche de Pisidie ${ }^{2}$.

Ce texte fameux a fait l'objet d'une erreur d'interprétation globale, due au fait que jusqu'à la Guerre de 14-18, on ne le connaissait que sous une version, celle d'Ankara, à laquelle s'ajoutaient quelques petits fragments d'Apollonie de Pisidie. Th. Mommsen ${ }^{3}$ a fait son édition et son commentaire à partir de l'inscription d'Ankara, et d'ailleurs nous parlons encore aujourd'hui du Monument d'Ancyre comme s'il s'agissait d'un document isolé.

1 W.M. Ramsay, Klio 22, 1929, 172.

2 Voir J. Scheid, Res Gestae Diui Augusti. Hauts Faits du Divin Auguste, Paris, Les Belles Lettres 2007, introduction, pour la description et les caractéristiques des inscriptions.

3 Th. Mommsen, Res gestae divi Augusti, Berlin, $1883^{2}$; ID., dans CIL III, p.769-799. 
L'édition de Mommsen est magistrale, même s'il ne connaissait pas la version d'Antioche et les fragments importants d'Apollonia, et même si le principat n'avait pas fait l'objet de la même quantité de recherches qu'aujourd'hui. Sa présentation est sobre. Il admet que le texte d'Ankara reproduisait celui de Rome, et comme il figurait sur les parois du temple d'Auguste et de Rome, certains, comme U. von WilamowitzMoellendorff ${ }^{4}$, ont commencé à investir trop dans la version d'Ankara.

Les choses ont changé en 1927 avec la découverte, à Antioche de Pisidie, d'une version complète, mais fortement mutilée, des Res Gestae en latin et, en même temps à peu près, d'autres fragments de la version de Pisidie ${ }^{5}$. Alors que la preuve était faite par ces documents que les Res Gestae de Galatie ne reproduisaient pas de façon exacte la disposition du texte affiché à Rome, puisque différents types de mise en page étaient désormais attestés, les éditeurs et commentateurs continuaient malgré tout à suivre la voie tracée par Mommsen. Cette attitude conduisit à une erreur quant à l'inscription d'Ankara, et une surinterprétation du document lui-même.

C'est notamment le livre de Wilhelm Weber sur Princeps. Studien zur Geschichte des Augustus, publié en $1936^{6}$, qui illustre ce fait. Induit en erreur par son orientation idéologique, Weber donna une interprétation générale inacceptable du texte, et il détermina en outre, par les corrections de texte qu'il proposait et par le style elliptique des très abondantes notes du livre, d'autres erreurs.

Même s'il précise que l'essentiel de son livre était rédigé en 1925, mais ne put être publié à cette date en raison de la situation catastrophique des maisons d'édition, et en dépit de l'énorme érudition contenue dans les riches notes où Weber a déversé un trésor de remarques sur tous les aspects des Res Gestae et même sur les institutions et sur l'historiographie augustéenne et impériale, son Princeps défendait une thèse inspirée des événements contemporains. W. Weber renouait, en effet, avec une interprétation

4 U. von Wilamowitz-MoellendorfF, "Res Gestae Divi Augusti”, dans ID. Kleine Schriften. V, 1. Geschichte Epigraphik Archaeologie, Berlin, 1937, 267-271.

5 W.M. Ramsay, A. von Premerstein, Monumentum Antiochenum. Die neugefundene Aufzeichnung der Res Gestae Divi Augusti im Pisidischen Antiochia (Klio Beiheft XIX), Leipzig, 1927. Pour les photographies de cette version, voir TH. Drew-BEar, J. Scheid, "La copie des Res Gestae d'Antioche de Pisidie", dans Zeitschrift für Papyrologie und Epigraphik 154, 2005, 217-260; W.H. Buckler, W.M. Calder, W.K. C., Guthrie, Monuments and Documents from Eastern Asia and Western Galatia, Manchester 1933, 51-52; Th. Drew-Bear, J. Scheid, "Les fragments des Res Gestae découverts à Apollonia de Pisidie", dans G. PACI (éd.), Contributi all'epigrafia d'età augustea (XIIr Rencontre d'épigraphie du monde romain, Macerata 9-11 settembre 2005), Macerata 2007, 433-448.

6 W. Weber, Princeps. Studien zur Geschichte des Augustus. I, Stuttgart-Berlin 1936 (le tome II n'a jamais paru). 
des Res Gestae qu'il avait partiellement reprise à Ulrich von Wilamowitz-Moellendorf. Depuis longtemps, les historiens tentaient de définir le genre auquel appartenaient les Res Gestae. Th. Mommsen ${ }^{7}$ les avait qualifiées de Rechenschaftsbericht, de "Compte rendu ", sans pouvoir entièrement imposer cette définition. À la même époque, Eugen Bormann ${ }^{8}$ avait proposé de les considérer comme une inscription funéraire associée au Mausolée, ou plutôt comme un éloge funéraire. Cette doctrine fut combattue avec succès par E. Kornemann ${ }^{9}$, et personne n'y revient depuis lors. D'autres savants ${ }^{10}$ ont invoqué l'influence hellénistique des Tatenberichte de souverains, de la description de hauts faits, un genre épigraphique attesté de Darius à Antiochos $1^{\text {er }}$ de Commagène. Mais là encore, on a pu montrer que le monde méditerranéen, même grec, était trop ignorant des coutumes orientales pour pouvoir s'en inspirer. Les analogies éventuelles doivent être considérées comme des coïncidences dues à la matière de ces textes plutôt que comme des emprunts ou des influences. On considérera donc les Res Gestae comme une auto-représentation épigraphique, comme une chronique des actes accomplis, des honneurs reçus et des générosités offertes au service de la république, bref comme une autobiographie d'un style particulier, qui est en relation avec les éloges triomphaux et funéraires des Romains ${ }^{11}$.

À tout cela Ulrich von Wilamowitz ${ }^{12}$ avait ajouté, contre l'avis de Mommsen, une idée originale, en comparant les Res Gestae à l'inscription que l'Empereur Hadrien fit dresser de son vivant au Panthéon d'Athènes. Wilamowitz y voyait une justification de son apothéose prochaine par un Auguste vieillissant. W. Weber a repris cette idée. Il attirait l'attention sur la richesse du vocabulaire de ce texte, qui emploie rarement deux fois le même terme, et l'habileté de sa construction. Les comptages de mots auxquels il se livre dans son édition avaient pour mission de démontrer les qualités artistiques de cette construction (fig. 1). Ainsi comptait-il le nombre de lignes suivants dans les deux parties de la version latine du Monument d'Ancyre, disposées à gauche et à droite de la porte d'entrée du temple:

$1^{\mathrm{e}}$ colonne $=46$ lignes

$4^{\mathrm{e}}$ colonne $=54$ lignes

$2^{\mathrm{e}}$ colonne $=46$ lignes

$5^{\mathrm{e}}$ colonne $=54$ lignes

$3^{\mathrm{e}}$ colonne $=43$ lignes

$6^{\mathrm{e}}$ colonne $=28$ lignes

7 Th. Mommsen, "Der Rechenschaftsbericht des Augustus" (1887), dans ID., Gesammelte Schriften. IV, Berlin 1906, 247-258.

8 E. Bormann, "Veranlassung und Zweck des sogenannten Monumentum Ancyranum », dans E. OeHLey (éd.), Verhandlungen der dreiundvierzigsten Versammlung deutscher Philologen und Schulmänner in Köln, Leipzig 1896, 180-193.

9 E. Kornemann, Mausoleum und Tatenbericht des Augustus, Berlin-Leipzig 1921, 12-18.

10 Mommsen 1887, 248 suiv.; A. Momigliano, Lo sviluppo della biografia greca (1971), Turin 1974, 76.

11 Voir pour tout ceci J. SCHEID, Res Gestae..., XXXVI-XLIII.

12 U. von Wilamowitz-MoellendorfF, "Res Gestae...", 267-271. 
En tout 135 lignes à gauche, 136 à droite, ce qui traduit, d'après Weber, l'imitation des deux stèles de Rome. Le nombre des lignes de la version grecque n'a apparemment rien à voir avec celui de la version latine:

$1^{\mathrm{e}}, 2^{\mathrm{e}}, 3^{\mathrm{e}}, 4^{\mathrm{e}}, 6^{\mathrm{e}}$ colonne $=23$ lignes

$5^{\mathrm{e}}$ colonne $=22$ lignes

$19^{\mathrm{e}}$ colonne $=11$ lignes

autres colonnes $=24$ lignes.

\begin{tabular}{|c|c|c|c|c|}
\hline Kol. & Zeilen & Kapite & & Worte \\
\hline Rg.: I & 46 & 7 & & 429 \\
\hline II/III $(46,43)$ & 89 & 11 & $(423,373)$ & ) 796 \\
\hline I/III & 135 & 18 & & 1225 \\
\hline IV & 54 & 6 & & 504 \\
\hline V/VI $1-28$ & $(54,28) 82$ & 11 & $(528,234)$ & 782 \\
\hline IV/VI 28 & 136 & 17 & & 1266 \\
\hline Insgesamt: & 271 & 35 & & 2491 \\
\hline Summa: VI $29-43$ & 15 & 4 & & 103 \\
\hline Gesamtsumme: & 286 & $35+4$ & & 2594 \\
\hline
\end{tabular}

Fig. 1. Exemple des comptages de mots effectués par Weber sur le texte d'Ankara.

Weber prévoyait la résistance de ses collègues à ce type de jeux, qui dépend entièrement des restitutions du texte. Dès l'une des premières notes de son commentaire, qui concernait les séquences de caractères dans le premier chapitre des Res Gestae, dans lesquels il découvrait un équilibre qu'il prétendait retrouver également dans la version grecque, il se justifiait en ces termes ${ }^{13}$ :

13 Weber, Princeps, note 548: Ich rechne damit, daß viele diese Tatsachen als völlig phantastische, überflüssige Spielerei ablehnen werden. Sie werden auch durch die Fülle weiterer Beobachtungen nicht belehrt werden, können darum leichter bei dem Dogma von der Erkennbarkeit der Schichten und Redaktionen bleiben. Wem es um den Gedanken ernst ist, einen Menschen des Südens, einer Welt, die vom Klang des Wortes, der Musik eines Satzes, der Magie der Form weiß, ganz zu erfassen, der wird leicht den Vorgang zu ergründen und zu begründen wissen. Man kann ihn bei Tacitus [...] wie in den Gratulationsbriefen des Plinius an Traian (1; 14), in den Hymnen des Isis-buches der Metamorphosen des Apuleius (XI), in den Kaiserhymnen bei Philo leg. Ad Gai. [...], in zablreichen anderen Prosastücken aus rhetorischer, enkomiastischer, hymnisch-liturgischer Sphäre (auch des jungen Christentums wie dem Apostolikum und gerade in überraschender Prägnanz und Fülle), rückwärtsgehend aber bis zu den altsumerischen oder ägyptischen Gebeten, Königsinschriften und Literaturstücken finden. Mir scheint es weiterer Hinweise zunächst nicht zu bedürfen. 
" Je prévois que beaucoup rejetteront ce fait comme un jeu inutile. Ces personnes ne seront pas non plus convaincues par la masse des observations contenues dans les pages suivantes, et peuvent ainsi s'en tenir d'autant plus facilement au dogme de l'identification de couches et de rédactions diverses [allusion à Kornemann]. Mais celui qui s'applique sérieusement à vouloir saisir dans sa totalité un homme du Sud [il s'agit d'Auguste], et un monde qui connaît la mélodie du mot, la musique de la phrase, la magie de la forme, saura aisément découvrir et justifier ce procédé. On le retrouvera aussi bien chez Tacite [...] que dans les lettres de félicitation de Pline à Trajan [...], dans les hymnes du livre d'Isis dans les Métamorphoses d'Apulée, dans les hymnes impériaux chez Philon, Leg(atio) ad Gaium [...], on le trouvera dans les nombreuses autres pièces de prose provenant des sphères rhétorique, encomiastique ou hymnico-liturgique (même dans le christianisme primitif et les écrits apostoliques, et ceci notamment avec une force et une abondance surprenante), ou encore en reculant dans le passé jusqu'aux prières, aux inscriptions royales et à la littérature du monde sumérien ancien et de l'Égypte. Il me semble que je n'ai pas à donner d'autres références.»

Le passage dont le commentaire appelle ce plaidoyer met précisément en évidence le côté aléatoire de ces jeux. La quatrième phrase du premier chapitre détruit la théorie de l'équilibre, car à 18 mots en trois propositions correspondent, dans la traduction grecque, 19 mots en cinq propositions. Or la traduction grecque est censée d'après Weber reproduire l'équilibre du texte latin. Il justifie cette divergence comme insignifiante, puisque le traducteur aurait choisi de donner une périphrase explicative du terme IIIuir rei publicae constituendae ${ }^{14}$. S'il avait été toutefois à tel point préoccupé de rendre la prose latine d'Auguste, le traducteur aurait pu chercher un terme grec plus court, surtout dans l'ouverture du texte, pour retrouver le prétendu équilibre. Bref, comme d'autres historiens l'ont souligné, les nombreux, "quasi, à peu près, environ, presque ", et dans le passage cité "de toute évidence " (offensichtlich), que Weber emploie partout cachent les difficultés de cette théorie, qui n'aurait de sens que si elle fonctionnait toujours.

Autre exemple, le titre même de la version grecque d'Ankara. Weber attirait l'attention sur le fait dans le titre de la version grecque d'Ankara, le mot theos se trouve placé au centre du titre et de l'inscription ${ }^{15}$. Reliant les deux parties des Res Gestae, initialement prévues pour être inscrites chacune sur un pilier, ce terme donnait pour ainsi dire son unité et sa signification à l'ensemble. La construction Sebastou theou (divin Auguste, au lieu de theou Sebastou) serait d'après Weber très rare et aurait été destinée à obtenir une construction qui placerait les deux syllabes significatives entre deux unités

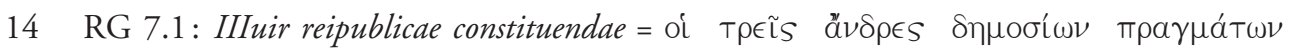

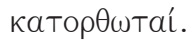

15 RG titre de la traduction grecque (Ankara): Methêrmêneuménai hupergráphêsan práxeis te kaì dôreaì Sebastồ theồ, hàs apélipen epì Rômê enkekharagménas khalkaîs stêlais dusin. On aurait donc 21 syllabes-theou- 21 syllabes. 
de 21 syllabes. Il est vrai que la formule Sebastou theou est moins fréquente que theou Sebastou, mais quelle conclusion tirer de cette constatation? Elle était possible, et peut donc difficilement révéler une intention profonde du rédacteur. D'autre part, il n'est pas du tout prouvé que ces deux syllabes étaient placées au milieu de deux séquences de 21 syllabes, comme le prétend Weber. L'inscription comporte dix-neuf colonnes et le terme theou est placé au-dessus de la neuvième colonne, ce qui correspond mal à l'idée qu'il est censé relier les colonnes des deux parties du texte. Qu'à cela ne tienne. Weber considérait que le texte devait initialement comporter dix-huit colonnes, mais qu'une dix-neuvième colonne de texte y a été finalement ajoutée, ce qui a entraîné le déplacement des colonnes, de telle sorte que theou ne se trouve plus à son emplacement stratégique au milieu des deux séquences de colonnes. Il est inutile d'aller plus loin, car on relève tout de suite les exagérations auxquelles la défense enthousiaste de sa théorie a entraîné Weber. Ce n'est pas avec ce type d'arguments que l'on peut faire accepter une reconstruction de l'inscription de Rome et toute la théorie quil l'accompagne.

Les comptages des lignes, des mots et même des caractères permettaient à Weber d'affirmer que les Res gestae étaient composées avec un art raffiné, et qu'elles constituaient une création artistique, une poièsis au plus haut degré. Cette composition poétique, équilibrée, proche des discours ciselés d'un Isocrate, trahirait la tension puissante entre un exposé rationnel, clair et simple des faits, et la volonté de composer un hieros logos, autrement dit le mythe de celui qui deviendrait ou était devenu un dieu. Dans son enthousiasme, Weber reconnait dans cette ouvre toute la force d'un vieillard qui, tel Michel-Ange construisant la coupole de Saint-Pierre, aurait su donner une dernière fois une image totale et parfaite de lui-même, de ses vertus et de son œuvre. Auguste aurait rédigé le document entre le début de sa dernière puissance tribunicienne, soit le 27 juin 14, et son départ de Rome, en juillet 14. Dans cette créativité, rapprochée de celle de Michel-Ange et de Bismarck âgés, Auguste se serait occupé même des petits détails, jusqu'à la disposition de l'inscription sur les deux colonnes et l'emplacement des mots et des phrases essentielles ou les indications pour les copistes futurs.

Les Res Gestae étaient donc d'après Weber une ouvre littéraire d'une grande puissance, que le Sénat aurait même fait inscrire dans tous les lieux de culte du Divin Auguste, du moins dans les cités qui auraient reçu des bienfaits d'Auguste. Le texte aurait été lu pendant les fêtes et célébrations, et aurait représenté l'histoire sacrée du dieu vénéré. Les temples d'Auguste seraient en quelque sorte l'image exacte du mausolée de Rome. Cette affirmation gratuite trouvait un reflet dans un stemma de transmission de l'archétype, qui est d'une complexité aussi rare qu'inutile (fig. 2). La dernière affirmation me paraît particulièrement absurde. Car le Mausolée d'Auguste n'était pas seulement la tombe d'Auguste, mais depuis longtemps celle de toute sa famille, dont il était alors le premier et le seul à être divinisé, et d'autre part parce qu'en général les temples d'Auguste, notamment en Asie Mineure et plus particulièrement à Ankara, étaient des temples de Rome et d'Auguste. Enfin, à Rome même le temple du Divin Auguste ne fut construit qu'au début du principat de Caligula. L'exemplaire des Res Gestae destiné au Temple d'Auguste à Rome (T) n'existait donc pas. 


\section{St e m ma:}

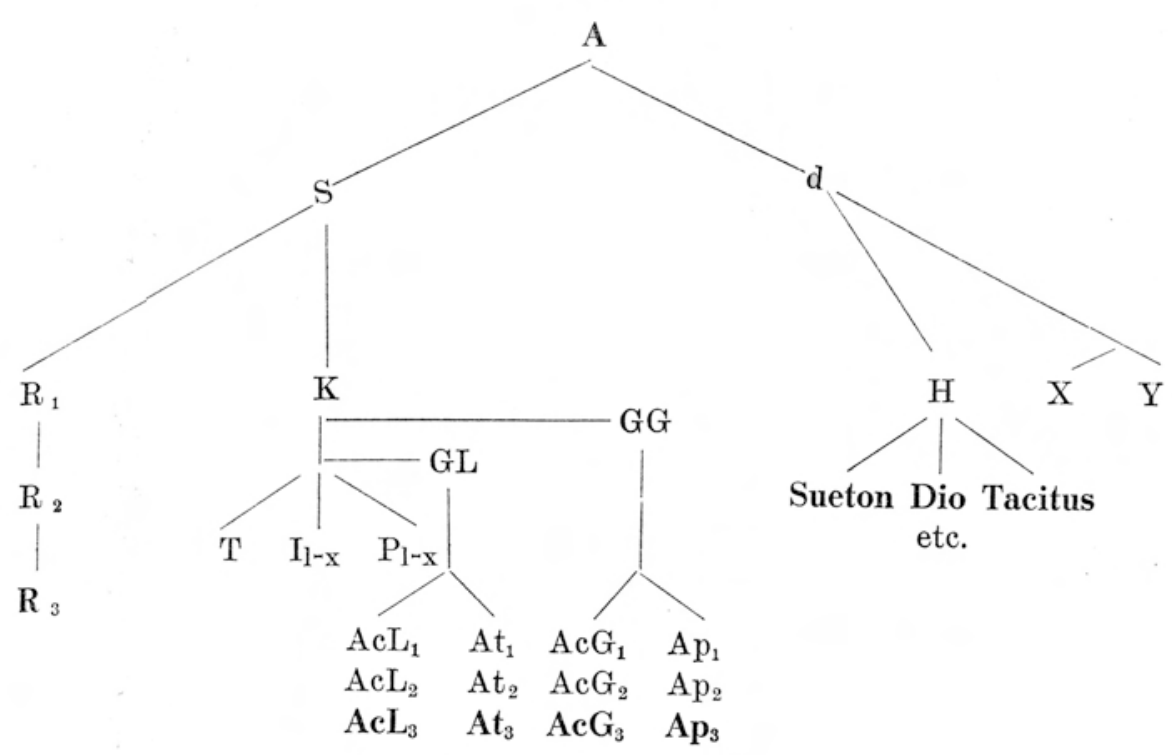

Fig. 2. Stemma des Res Gestae d'après Weber (A = archétype, texte d'Auguste, $S$ = exemplaire du Sénat, $\mathrm{R} 1$ = copie pour le sculpteur, $\mathrm{R} 2$ autres copies nécessaires,

R3 inscription du mausolée, $K=$ chancellerie, $G=$ Galatie, $T=$ temple d'Aug. à Rome, I1-x = copies pour l'Italie, $\mathrm{P} 1-\mathrm{x}=$ copies pour les provinces, $\mathrm{H}=$ copie de la tradition historique, $\mathrm{XY}$ copies privées).

Toutes ces spéculations et théories erronées étaient dues à l'idéologie de l'Allemagne contemporaine. Ce n'est pas pour des raisons archéologiques, épigraphiques et historiques que W. Weber conférait à l'inscription d'Ankara une aura exceptionnelle. L'inscription aurait été en fait par son style et par sa mise en page une œuvre d'art totale, réalisée par un chef exceptionnel. L'harmonie claire et sobre du texte constituerait, d'après Weber, une parfaite représentation des tensions intérieures d'Auguste, et devrait être rapprochée de ses portraits. Les Res Gestae combineraient la clarté de la raison et de la puissance de la volonté. En elle se ferait l'alliance de l'ordre le plus strict et de la passion parfaitement dominée. Dans les phrases et jusque dans l'élégance nerveuse des caractères " étroits et allongés " utilisés dans la version latine des Res Gestae d'Ankara, Weber reconnaît l'expression de la pulsion d'une force intérieure et le règne de la volonté (Auftrieb innerer Kräfte und... Herrschaft des Willens ${ }^{16}$ ). On n'est pas loin du 
" triomphe de la volonté », et de la représentation de l'homme d'État exceptionnel qui serait aussi un grand artiste.

Une telle glorification de l'inscription d'Ankara est bien entendu absurde, et surtout elle n'avait plus de sens à l'époque de Weber. Si Th. Mommsen pouvait travailler sur la mise en page du texte d'Ankara, c'est parce que c'était à son époque le seul texte qui était connu, à l'exception de quelques lignes du texte grec d'Apollonie de Pisidie. Telle n'était plus la situation dans les années 1920 et 1930. La surévaluation de la copie d'Ankara était erronée dès le départ. L'inscription a été mise en page en fonction de la paroi du temple sur laquelle elle a été gravée.

Il convient donc de se méfier des comptages de mots et de caractères ainsi que des interprétations trop enthousiastes de Weber, qui tendent même à restituer le texte en fonction de ces idées, et peuvent susciter de nouvelles erreurs de détail. À cela s'ajoute le fait que Weber avait vérifié en 1929 le texte des Res gestae sur les moulages du monument d'Ankara réalisés en son temps pour Th. Mommsen et conservés à Berlin dans l'Altes Museum. Cette vérification donna ensuite lieu à une reconstruction du texte qui était extrêmement compliquée (fig. 3). Ainsi, nombre d'éditions actuelles charrient des traces des interventions de W. Weber, qui toutes découlent de la surévaluation de l'inscription latine d'Ankara. Les exemples les plus évidents de cette influence sont constitués par les éditions de J. Gagé et de H. Volkmann ${ }^{17}$. L'édition de J. Gagé parut un an avant que le livre de Weber, mais ce dernier avait vu les épreuves de l'ouvrage et avait indiqué à Gagé ses révisions du texte. Aussi Gagé, influencé par cette volonté de remonter à l'archétype de Rome à travers la copie d'Ankara, imagina-t-il un système d'impression très compliqué pour représenter à travers son texte le modèle de l'inscription d'Ankara (fig. 4). Les différentes polices et les signes diacritiques, qui étaient différents de ceux que les épigraphistes utilisaient, renvoyaient soit au texte d'Ankara, quand il était conservé, soit à celui des autres versions, en précisant si le texte était préservé ou non sur le monument d'Ankara. En outre la linaison était celle de l'inscription d'Ankara. Cette présentation du texte de l'inscription fut aussi celle de H. Volkmann ${ }^{18}$ (fig. 5), suivie en 1944 par E. Malcovati ${ }^{19}$. Cette présentation du texte n'est plus attestée dans les éditions postérieures, notamment S. Riccobono, G. Pugliese Carratelli, E. Weber et

17 J. Gagé, Res Gestae Divi Augusti ex monumentis Ancyrano et Antiocheno Latinis, Ancyrano et Apollonensi Graecis, Paris 1935.

18 H. Volkmann, Res gestae divi Augusti. Das Monumentum Ancyranum (1942, fasc. 29-30 des Kleine Texte für Vorlesungen und Übungen), Berlin.

19 E. Malcovati, Caesaris Augusti Imperatoris operum fragmenta (Corpus Scriptorum Latinorum Paravianum, 38), Turin 19211/19445. 
G. G. Belloni, qui donne une édition conforme aux principes épigraphiques ${ }^{20}$. Comme le volume de Gagé, celui de Volkmann connut de nombreuses rééditions. Or rien ne justifiait plus cette référence au monument d'Ancyre. Ainsi, une vision idéologique du document inspira-t-elle des erreurs d'appréciation, des restitutions difficiles à accepter et une présentation absurde du texte dans l'édition de référence.

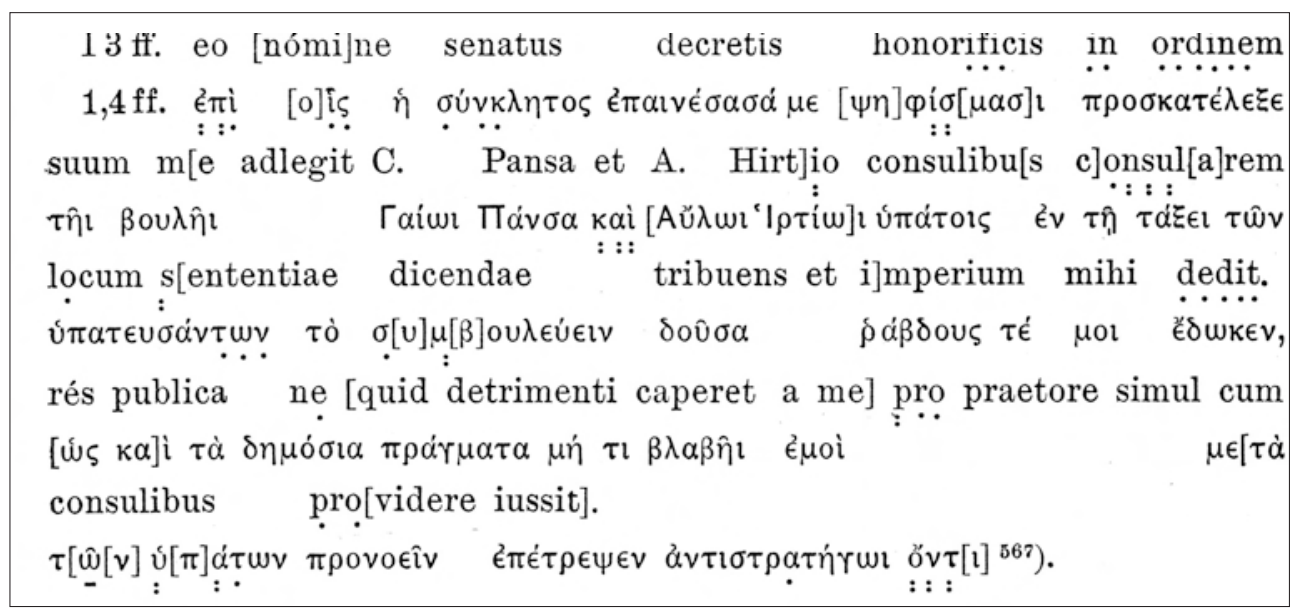

Fig. 3. Exemple du texte restitué de W. Weber ( $1^{\text {re }}$ colonne, 1.3 et 1.4 des copies latine et grecque d'Ankara).

Des vérifications systématiques à l'aide des photos, excellentes, de la fin du XIX ${ }^{\mathrm{e}}$., permettent d'éliminer la plupart des prétendues leçons de Weber, et le travail de St. Mitchell sur le monument même confirme que si le texte actuel est parfois mieux ou moins bien lisible que sur les anciennes photos, il correspond à celui que j’ai proposé dans mon édition.

Deux exemples peuvent illustrer les erreurs dues au travail de W. Weber, la restitution d'un passage du chapitre 10, qui dépend du portrait d'Auguste qu'il voulait tracer, et une ligne du chapitre 9, où le style elliptique de Weber a induit en erreur un certain nombre de ses successeurs.

20 S. Riccobono, Acta Divi Augusti. Pars prior. Res Gestae Divi Augusti ex Monumentis Ancyrano, Antiocheno, Apolloniensi, graece et latine, Rome 1945. Riccobono adopte encore la linaison d'Ankara; G. Pugliese Carratelli, Imperator Caesar Augustus. Index rerum a se gestarum. Testo latino e versione greca secondo gli esemplari Ancirano Antiocheno Apolloniense, Florence 1947; G.G. Belloni, Le Res gestae Divi Augusti, Milan 1987; E. Weber, Meine Taten. Res Gestae Divi Augusti nach dem Monumentum Ancyranum, Apolloniense und Antiochenum, Düsseldorf-Zurich $1999^{6}$. 


\section{GAGÉ 1935}

\section{RES GESTAE DIVI AVGVSTI, I $\quad[I, 1-9]$}

Anc. 1. 1 Annós undéuiginti natus exercitum priuáto con- Ant.

1 silio et privatá impensá I comparáui, [\$] per quem rem I publicam [á do]minatione factionis oppressam I

1. 1 a domi[nat-] Ant.; a om. priores II

[I, 1-14]

RES GESTAE DIVI AVGVSTI, I

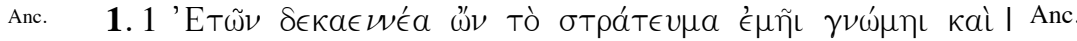

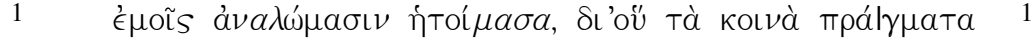

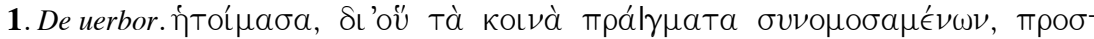

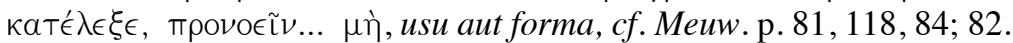

Fig. 4. Exemple du texte dans l'édition de J. Gagé (Caractères droits $=$ conservé sur le Monument d'Ankara ; caract. gras = conservé [aussi] à Apollonia et à Antioche ; caractères gras entre crochets $=$ seulement conservés à Antioche ; italique $=$ absent partout $)$.

\section{VOLKMANN 1942}

I c. 1 .

(Ant. I)

1 Annós undèviginti natus exercitum priváto consilio et privatá impensá

2 comparávi, [§] per quem rem publicam á dominatione factionis oppressam

c. I I.

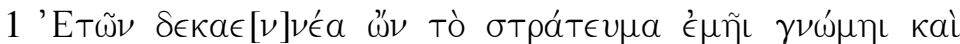

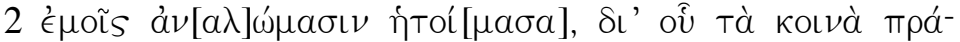

Fig. 5. Exemple du texte dans l'édition de H. Volkmann (caractères droits $=$ conservé à Ankara $;$ italiques $=$ Ankara-Antioche-Apollonia ; italiques soulignées $=$ Antioche et Apollonie ; [ ] lacune partout). 
Le chapitre 10 des Res Gestae offre un bel exemple des dangers que représentent ces spéculations. Dans la $2^{\mathrm{e}}$ colonne du texte latin de Ankara on lit, à la ligne $22^{21}$ :

[--- q]VOA[d] VIVEREM.TRIBVNICIA.POTESTAS.MIHI

[--- . 2. Pontif] EX MAXIMVS Ne FIEREM IN VIVI [C]ONLE-

[gi ---],

c'est-à-dire " que, tant que je vivrai, la puissance tribunicienne me soit " (lacune), puis, après la lacune, «afin que je ne sois pas élu grand-pontife à la place d'un collègue vivant ". Le problème tourne autour de la restitution de la lacune qui évoque l'attribution de la puissance tribunicienne. Cette formule est importante, car l'appréciation juridique de l'acte et de la fonction en dépendent.

Laissant vacantes certaines lacunes du texte, Mommsen avait restitué le passage de la manière suivante: ... Et sacrosan/[ctus ut essem... et ut q]uoa[d] uiuerem, tribunicia potestas mihi I [esset, lege sanctum est]. Les fragments de Antioche, col. III, 1. 30 suiv., ont permis en 1927 à Ramsay-von Premerstein ${ }^{22}$ de présenter un texte plus complet: [sacrosa] NCTV[s in perp]ETVM [ut essem et, quo]AD [uiuerem tribunicia pot]IE[stas mibi] $T[$ ribueretu]R, ST[atutum est. 2. Pontifex maximus ne fier]EM..., "il fut décidé que je sois sacrosaint à jamais, et que tant que je vivrais, la puissance tribunicienne me soit attribuée. 2. Afin que je ne sois pas élu grand-pontife etc. "

La nouveauté des nouveaux fragments et de cette restitution consiste dans la description de la procédure d'attribution de la puissance tribunicienne. Comme le texte grec indiquait que ces mesures étaient accordées en vertu d'une loi: $\mathrm{NO}[\mu \omega \mathrm{L} \quad \grave{\epsilon} \kappa]$ $\Upsilon \mathrm{P} \Omega \Theta \mathrm{H}$ (Ankara col. 5, 1.19), il est préférable de supposer que le texte mentionnait une loi. Ramsay et von Premerstein supposaient que statutum est suffit pour décrire la procédure législative. Reprenant le problème, W. Weber ${ }^{23}$ lit sur le fragm. 10d d'Antioche, à la place de $[\ldots] \mathrm{R}$ ST[...], c'est-à-dire pour le R final de T[ribueretu]R et les deux premières lettres de $S T$ [atutum], plutôt $M$ que R, et A plutôt que $T$, comme le premier éditeur du fragment, Reid, l'avait déjà fait en 1916. Et donc Weber restitue: [sacrosa] NCTV[s in perp] ETVM [ut essem, quo]AD [uiuerem tribu!nicia pot] IE[stas mihi esset per lege]M SA[nctum est. 2. Pontifex maximus ne fier]EM... Un regard sur la photographie du fragment d'Antioche (fig. 6) montre toutefois qu'il ne peut y avoir de $A$ après le $S$. Il peut y avoir discussion pour le premier caractère dont un vestige légèrement arrondi est conservé, et qui peut être, comme Ramsay-Premerstein le reconnaissent eux-mêmes, un $\mathrm{A}$, un $\mathrm{M}$ ou un $\mathrm{R}$, mais il est impossible de lire SA[nctum].

21 Weber, Princeps, 176*.

22 W.M. Ramsay, A. von Premerstein, Monumentum Antiochenum. Die neugefundene Aufzeichnung der Res Gestae Divi Augusti im Pisidischen Antiochia (Klio Beiheft XIX), Leipzig, 1927. Pour les photographies de cette version, voir Drew-BEAR, ScheID, "La copie des Res Gestae d'Antioche...", 217-260.

Weber, Princeps $131^{*}$. 
Convaincu par sa leçon, Weber insère dans la lacune de la copie d'Ankara le texte qu'il a cru lire sur le fragment d'Antioche:

22 [ctus in perpetuum ut essem, q]VOAD VIVEREM.TRIBVNICIA.POTESTÁS.MIHI

23 [esset, per legem sanctum est. 2. Pontif] EX MaXimVS Ne FIEREM IN VÍvi [c] ONLE-

24 [gi ---].

Weber prétend même avoir lu ESS[et] au début de la ligne 23 d'Ankara, ce qui est impossible vu l'état de la pierre. Il est en tout cas certain que le texte d'Antioche comporte ST[atutum, mais pour le $\mathrm{R}$ et d'ailleurs la trace du $\mathrm{T}$ initial de tribueretur, qui est peu visible sur les photographies, on peut avoir des doutes. Ce jeu avec les restitutions, qui permettent d'ajouter ou de supprimer des caractères, démontre à quel point la théorie de la structure prétendument artistique du texte et de son équilibre parfait dépend du remplissage des lacunes. Et bien entendu, le portrait même d'Auguste découle de certaines restitutions. On en a un exemple au chapitre 2, qui évoque la vengeance de l'assassinat de César et la très relative clémence d'Auguste ${ }^{24}$. Au prix de restitutions diverses Weber y gomme un élément désagréable du portrait de son héros. On peut, certes, souligner que la leçon qui fait l'unanimité noircit ce portrait, mais elle a l'avantage d'être en accord avec la tradition sur la cruauté d'Octavien pendant les Guerres civiles, une brutalité que le vieil Auguste et son entourage ne niaient nullement.

W. Weber avait entrepris en 1929 un contrôle du texte des copies d'Ankara à l'aide du moulage des inscriptions. Contrairement à ce qu'il avait affirmé à J. Gagé, son texte révisé ne figure pas dans une annexe de son Princeps, mais dans les notes du livre. Ces corrections ont souvent été adoptées sans critique par ses successeurs. Très souvent cependant, les leçons wébériennes sont illusoires, ainsi que W. Kolbe ${ }^{25}$ l'avait déjà souligné. Weber indiquait ses corrections

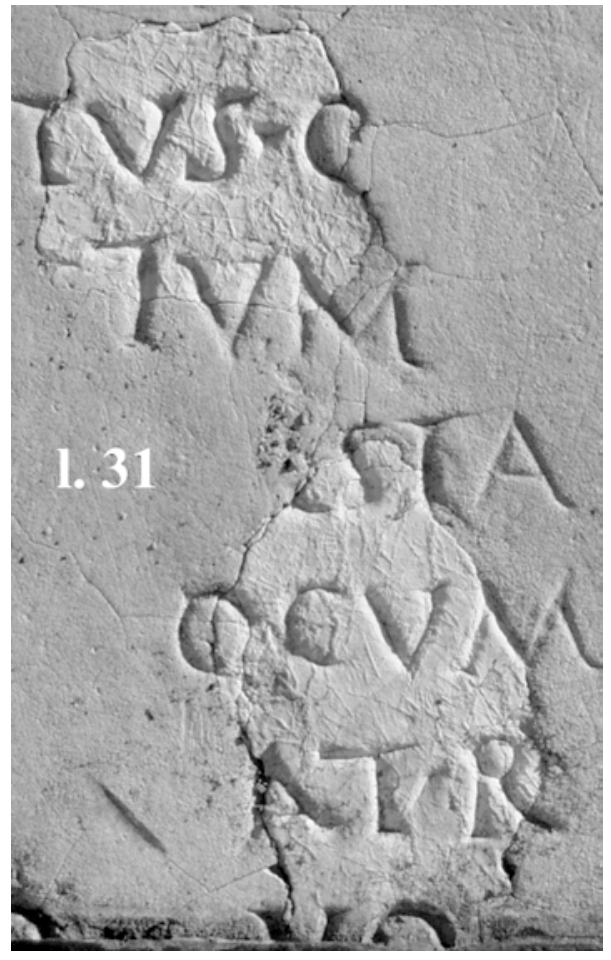

Fig. 6. Fragment 10 dge du fragment d'Antioche.

24 Voir ScheID, Res gestae, 30 suiv.; ID., "La “clémence” d'Auguste d'après les Res Gestae Divi Augusti» (sous presse).

25 W. Kolbe, "W. Weber, Princeps. Studien zur Geschichte des Augustus. I etc.", dans Göttingische Gelehrte Anzeigen 201, 1939, 152-169. 
au moyen d'un système très compliqué de signes diacritiques dont la catégorie " totalement incertain ", c'est-à-dire un caractère marqué de deux points, devrait d'emblée inviter à la prudence.

Puisque le style des notes du livre de W. Weber est très dense et elliptique, une source d'erreurs supplémentaire pouvait s'y ajouter. L'un des plus beaux exemples se trouve au chapitre 9.1, qui concerne des jeux célébrés tous les quatre ans (quinto quoque anno) pour le salut d'Auguste. Le texte latin, fortement mutilé, a été reconstruit d'après la version grecque du passage, qui est entièrement conservée. Même si, au fond, la restitution d'une lacune dans une inscription peut paraître un jeu un peu vain, c'est précisément sur un détail de cette restitution que porte le petit problème historique posé par ces jeux.

Le texte actuellement donné de ce passage, avec les compléments fournis par la version d'Antioche, est le suivant:

15 Vota $p[$ ro ualetudine mea susc]ipi p[er con]sules et sacerdotes qu[in] to qu[oque anno decreuit senatus. ex iis] uotis s[ae]pe fecerunt uiuo m[e ludos aliquotiens sacerdot]um quattuor amplissima collel[gia, aliquotiens consules].

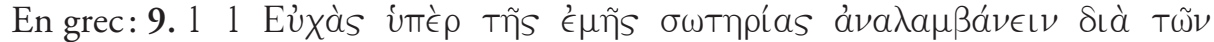

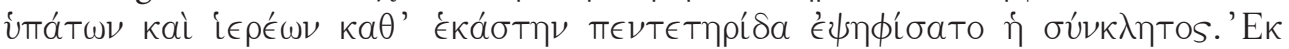

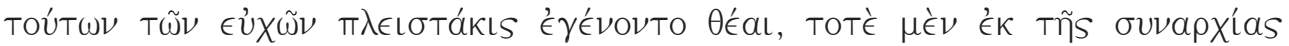

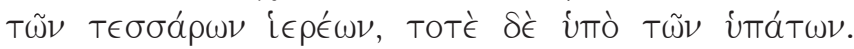

La restitution du texte latin remonte à Th. Mommsen qui a proposé pour le début du chapitre [uota pro ualetudine suscipi per cons] ules, parce que, à la ligne 20 du même chapitre, Auguste rappelle qu' "à titre privé et par municipes, tous les citoyens romains en plein accord et sans relâche ont fait des supplications pour (sa) santé dans tous les temples 26 ". Mommsen ignorait encore les fragments d'Antioche qui permettaient de restituer Vota p rro en début du chapitre. C'était aller vite en besogne, car valetudo

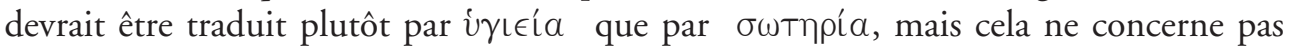
notre problème. Je renvoie pour cela à mon édition.

Dans les éditions postérieures au livre de Weber ce passage est présenté de façon

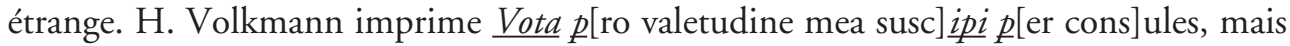
il met dans l'apparat critique: [vale]tudin[e] Weber [salute] Bergk, Weinstock. Weber aurait déchiffré sur le moulage de l'Antikes Museum cinq caractères du mot valetudine, alors que Bergk et Weinstock ont restitué salute dans une lacune. Par conséquent Volkmann retient la proposition de Weber, mais prudemment en pointant les lettres, car il doute, comme souvent, des leçons «très incertaines " de ce dernier. E. Malcovati ${ }^{27}$

26 RGDA II, 9, 2. [Pri]ua[ti]m etiam et municipatim uniuer[si] I [ciues unanimite]r con[tinente] $r$ apud omnia puluinaria pro uale ${ }^{20}[t u$ ]din[e mea s]upp[licauerunt], en grec: 2 Kai

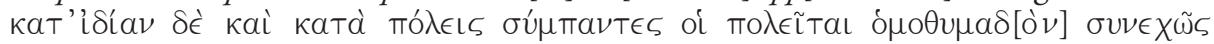

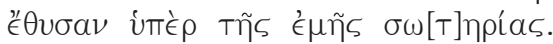

27 H. Malcovati, Imperatoris Caesaris Augusti operum fragmenta, Turin 19444, 118. 
et S. Riccobono ${ }^{28}$ proposent dans le texte: Vota p[ro ualetudine mea susc]ipi p[er cons] ules et signalent dans l'apparat critique: uota p[ro uale]tudin[e Weber ex Anc. vestigiis. Ils reprennent en fait le texte de Volkmann. Enfin, G. Pugliese Carratelli ${ }^{29}$ va plus loin, puisqu'il adopte dans le texte même la proposition de Weber: uota p[ro ualetu] $\operatorname{din}[\mathrm{e}]$, en signalant uniquement par les italiques que les lettres - tudin sont difficiles à déchiffrer.

Or, tout ceci est une erreur. Weber ${ }^{30}$ (fig. 7) a seulement précisé de façon laconique que, dans II, 9, 1, ligne 15, la restitution [pro ualetudine], entre crochets droits!,

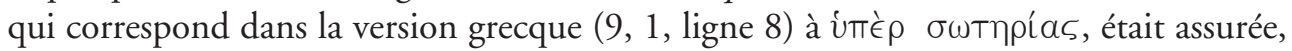
puisqu'elle pouvait s'appuyer sur le texte de II, 9, 2, lignes 19 suiv. [vers. lat.] = 9, 2, ligne 15 [vers. gr.]; dans ces lignes pro vale]tudin[e correspond effectivement à ímé $\rho$

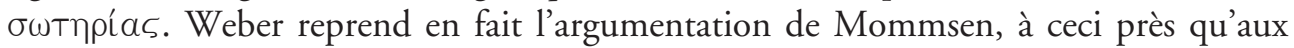
lignes 19-20, il croyait partiellement déchiffrer, très audacieusement comme toujours, pro valetudine. Il suffit de vérifier sur les photos et sur les moulages de Berlin, pour s'apercevoir que le début de la ligne 9, 1, 15 du fragment d'Ankara n'existe pas, et qu'il n'est pas question de le restituer. Volkmann et les trois autres auteurs nommés à sa suite ont mal compris la phrase de Weber, en confondant la ligne 15 avec la ligne $19 \mathrm{du}$ texte latin d'Ankara; cette erreur était d'autant plus facile que le passage correspondant à la 1.19 du texte latin était la $15^{\mathrm{e}}$ ligne du texte grec. Weber ne dit nulle part qu'il a déchiffré vale]tudin[e à la ligne 15 du texte latin, mais c'est à la ligne 19 qu'il propose cette leçon. Sa proposition est d'ailleurs étrange, car la version d'Ankara donne en fait ici pro vale[tudine], et non, comme Weber l'écrit [vale]tudine, celle d'Antioche [valetu] din [e]. On a l'impression que Weber a mélangé ses fiches et ce qu'il propose n'est en fait pas la leçon de la ligne 19 du texte d'Ankara, mais celui du fragment d'Antioche. Cette confusion n'a certainement pas facilité la compréhension de la note. Volkmann a en outre ajouté une deuxième erreur en considérant que les lettres Vota p[ro étaient aussi conservées à Ankara.

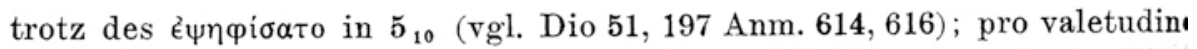

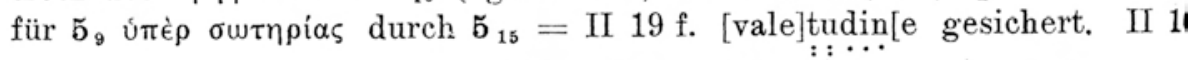

Fig. 7. Note 613 de Weber, Princeps, $174^{*}$

28 S. Riccobono, Acta Divi Augusti. I, Rome 1945, 30.

29 G. Pugliese CarRatelli, Imperator Caesar Augustus. Index rerum a se gestarum. Testo latino e versione greca secondo gli esemplari Ancirano Antiocheno Apolloniense, Florence 1947.

30 Weber, Princeps, $174^{*}$, note 613: "pro valetudine für 5 , intè $\sigma \omega T \eta p i ́ a s$ durch $5{ }_{15}=$ II $19 \mathrm{f}$. [vale] tudin[e gesichert ». 
J'arrête là. Malgré les avertissements d'un historien du début du XIX ${ }^{\mathrm{e}}$ s., $\mathrm{H}$. Heinen ${ }^{31}$, qui avait déjà réfuté la restitution de pro valetudine au début du chapitre 9.1, on continue jusqu'à ce jour de reproduire le texte cité, y compris Volkmann, qui met simplement en doute la lecture de Weber. Or celle-ci est impossible parce que sur l'inscription d'Ankara, ces lignes ont disparu ${ }^{32}$.

H.G. Pflaum, qui m'a appris l'épigraphie romaine, nous avertissait toujours que la pire faute dans l'édition et l'utilisation des sources directes n'était pas de se tromper, mais de créer des monstres. Ceux-ci ont ensuite la vie dure, d'autant plus dure qu'ils paraissent être fondés sur des sources directes. J'ai présenté deux monstres, un grand, la surinterprétation des Res Gestae par W. Weber, qui a bloqué les études pendant une génération, et un petit, tout simplement dû au fait que les auteurs du petit monstre ne connaissaient pas l'allemand et même s'ils le connaissaient, parce qu'ils ne vérifiaient pas l'inscription sur les photos ou les moulages. N'est-ce pas, en fin de compte, leur principale erreur?

John SCHEID

Professeur au Collège de France

john.scheid@college-de-france.fr

31 H. Heinen, "Zur Begründung des römischen Kaiserkultes. Chronologische Uebersicht von 48 v. bis 14 n. Chr.", dans Klio 11, 1911, 129-177.

32 J'ai pu moi-même vérifier cela dans le dépôt de l'Antikes Museum grâce à l'obligeance de Mme S. Brehme. 